\title{
Endocrine Disorders, Fertility and Sexual Health
}

\author{
Nina Salooja, Zeev Shoham, \\ and Jean-Hugues Dalle
}

Increasing numbers of HSCT are being performed annually and outcomes continue to improve. As a result, survivorship issues are assuming increasing importance. Chemotherapy and radiotherapy remain cornerstones of HSCT treatment, but whilst lifesaving, they threaten endocrine function, fertility and sexual function.

\subsection{Endocrine Function}

Underlying disease, pre-HSCT treatment, development of chronic GVHD and prolonged corticosteroid treatment can all contribute to the risks of endocrine dysfunction inherent from conditioning regimens used for HSCT. Systematic followup is important to identify and treat endocrine defects before clinical impact, and this is particularly important in children where growth and puberty are at risk.

\section{N. Salooja $(\bowtie)$}

Centre for Haematology, Imperial College,

London, UK

e-mail: nina.salooja@imperial.ac.uk

\section{Z. Shoham}

Department of Obstetrics and Gynecology, Kaplan Medical Center, Rehovot, Israel

J.-H. Dalle

Department of Pediatric Hematology-Immunology, Hospital Robert-Debré, Assistance Publique-

Hôpitaux de Paris, Paris Diderot University, Paris, France

\subsubsection{Thyroid Dysfunction}

\subsubsection{Background}

The most common abnormality of thyroid function after HSCT is primary compensated hypothyroidism. This may not require treatment and commonly resolves. Overt hypothyroidism may be primary or less commonly central. Other thyroid disorders include autoimmune thyroid disease (thyroiditis, Graves' disease) and thyroid cancers (carcinomas or benign adenomas). In a single-centre study of 791 patients followed up for 38 years, new cases of thyroid dysfunction continued for 28 years after HSCT highlighting the need for indefinite follow-up (Sanders et al. 2009).

Risk factors for hypothyroidism post-HSCT include the use of TBI with single dose being associated with a five to sixfold higher risk than fractionated TBI. BU-based regimens are more likely to cause thyroid problems than those containing CY only, and patients with malignant disease (e.g. Hodgkin lymphoma) are more likely to get thyroid dysfunction than patients with non-malignant diseases (e.g. aplastic anaemia). The age of the patient is also important with younger patients at higher risk. In relation to thyroid cancer, a retrospective study which included data on $>68000$ patients showed that the relative risk (RR) of thyroid cancer was approximately threefold higher following HSCT than in the general population. The RR was $>20$ if transplanted before the age of 10 years and close to 5 if transplanted between age 11 and 
20 years. Female gender and GVHD were additional risk factors (Cohen et al. 2007).

\subsubsection{Prevention/Management}

Patients should have annual laboratory assessment of thyroid function. Annual clinical examination should include palpation of the thyroid gland, and there should be a low threshold for arranging a thyroid ultrasound.

\subsubsection{Hypoadrenalism}

\subsubsection{Background}

The main risk factor is use of glucocorticoids which lead to central corticotrophin deficiency. Adult patients receiving $5 \mathrm{mg} /$ day prednisolone or more or paediatric patients receiving $>0.3-0.5 \mathrm{mg} / \mathrm{kg} / \mathrm{day}$ for more than 3 months are at risk. TBI can also cause corticotrophin deficiency as can drugs. Symptoms can be non-specific including fatigue, weakness, nausea, weight loss and hypotension. Some symptoms may mimic GVHD. Diagnosis requires paired morning cortisol and ACTH levels. If the results are nonconclusive, then additional investigations should be arranged with an endocrinologist.

\subsubsection{Prevention/Management}

When confirmed hydrocortisone should be given with additional doses to cover stresses such as illness, infection or surgery. Subsequently, regular evaluation is required as it may be possible to reduce/stop medication.

\subsubsection{Growth}

\subsubsection{Background}

Short stature is multifactorial after transplant. It is a recognized side effect of radiation to the hypothalamic-pituitary area given in childhood, as a result of a reduction in growth hormone (GH) secretion. Radiation can also induce bone lesions. Pre-transplant cranial radiation (e.g. patients with ALL) is also relevant, and singledose TBI rather than fractionated radiation increases the risk further.

Additional contributory factors to short stature in these patients include underlying disease (e.g.
Fanconi anaemia), other hormone deficiencies (including thyroid and gonadal hormones), nutritional deficits, illness, steroids and GVHD. Male sex and young age at time of transplant are additional risk factors.

\subsubsection{Prevention/Management}

Children's growth velocity should be closely monitored with height and weight documented at each clinic visit. A possible increased risk of secondary malignancies has been described in patients receiving GH therapy after previous neoplasia; this has raised concerns regarding the use of GH in the absence of sufficient long-term follow-up data. As a consequence of this, there are currently no clear guidelines for the use of $\mathrm{GH}$ in these patients. A paediatric endocrinologist should be involved if growth rate is abnormal based on bone age and pubertal stage (Chow et al. 2016) and the use of GH therapy discussed for severe growth retardation (-2SD).

\subsection{Gonadal Dysfunction and Infertility}

\subsubsection{Background}

Normal reproduction in both sexes requires germ cells and an intact hypothalamic-pituitary endocrine axis. In female patients the uterus must be both receptive to implantation and capable of undergoing growth during pregnancy. Chemotherapy and radiation can lead to damage in all of these areas and compromise the likelihood of successful parenthood after HSCT. Before starting any chemoradiotherapy regimen, the potential effects on the future fertility of the patient should be considered and discussed with the patient together with a discussion of fertility-preserving strategies.

\subsubsection{Gonadal Dysfunction in Women Following Chemoradiotherapy}

Women are born with a finite number of eggs which can be fertilized for pregnancy and depleted over time as a result of physiological apoptosis or 
else menstruation. Chemoradiotherapy depletes further the number of follicles by (1) activating apoptotic pathways, (2) causing fibrosis of stromal blood vessels, (3) activating resting (antral) follicles, leading to a 'burn-out' effect (Meirow and Nugent 2001; Kalich-Philosoph et al. 2013). The degree of ovarian damage is related to the dose and type of chemotherapeutic agent used, and baseline ovarian reserve which in turn is dependent on age and previous treatment. Manifestations of premature ovarian failure range from premature menopause to varying degrees of infertility. Alkylating agents have the highest ageadjusted odds ratio of ovarian failure (Meirow 2000). A combination of BU and CY is particularly gonadotoxic to females, but younger patients who receive $\mathrm{CY}$ only may have some gonadal function preserved and pregnancies following CY are well described (Salooja et al. 2001). TBI is also potentially sterilizing. The estimated median lethal dose of radiation for the human oocyte is less than 2 Gy (Wallace et al. 2003). The effective sterilizing dose (ESD) decreases with increasing age, and whilst estimated as $18.4 \mathrm{~Gy}$ at 10 years of age, the ESD is approximately $14.3 \mathrm{~Gy}$ at 30 years of age, and only $6 \mathrm{~Gy}$ in women over age 40 (Wallace et al. 2003).

\subsubsection{Gonadal Dysfunction in Men Following Chemoradiotherapy}

In male patients, spermatogenesis is impaired, but testosterone levels generally remain normal because of the relative resistance of testosterone producing Leydig cells to chemoradiotherapy. As a result, secondary sexual characteristics remain normal for male patients and typically testosterone levels and luteinizing hormone (LH) levels are in the normal range. Spermatogonia are very sensitive to irradiation, and it takes approximately 2 years for sperm counts to recover to preirradiation levels after a single dose of $1 \mathrm{~Gy}$ (Meistrich and van Beek 1990). With higher doses, azoospermia persists longer or may be permanent. Following HSCT conditioned with TBI, the majority of men will be azoospermic. Chemotherapy only regimens are also associated with azoospermia but to a lesser degree (Rovo et al. 2013). Following BU, for example, approximately $50 \%$ will be azoospermic, whilst after $\mathrm{CY}$ alone, recovery of spermatogenesis is more frequent.

\subsubsection{Uterine Dysfunction in Women After Radiation}

Uterine development commences at puberty and is associated with an increase in both size and vascularity (Laursen et al. 1996). Exposure to radiation leads to reduced vascularity, fibrosis and hormone-dependent endometrial insufficiency, which subsequently lead to adverse reproductive outcomes. Increased rates of infertility, miscarriage, preterm labour, intrauterine growth retardation and low newborn birth weight have been described (Reulen et al. 2009), particularly if conception occurred within a year of radiotherapy (Fenig et al. 2001).

\subsubsection{Prevention/Management of Gonadal Failure}

\subsubsection{Fertility Preservation in Males}

Sperm cryopreservation is an established fertility preservation option for post-pubertal boys and men. Sperm can be used either for artificial insemination or, if the quantity and/or quality of sperm are insufficient, for intracytoplasmic sperm injections for in vitro fertilization. There is a chance of sperm recovery with time particularly if the patient was under the age of 25 years at transplant, did not have TBI and has no evidence of chronic GVHD (Rovo et al. 2013). These patients require reassessment at intervals to ascertain their fertility potential.

\subsubsection{Fertility Preservation Techniques in Females}

\section{Gonadotropin-Releasing Hormone Agonists (GnRHa)}

Despite success in animal models, the value of GnRHa to preserve ovarian function during 
chemotherapy in human subjects is uncertain. A Cochrane database review (2011) concluded that the use of GnRH agonists should be considered for ovarian protection in women of reproductive age who are receiving chemotherapy (Chen et al. 2011).

\section{Embryo and Oocyte Cryopreservation}

Embryo and oocyte cryopreservation are preferred methods of fertility preservation in women who require sterilizing treatment. Use of donor embryos/oocytes can also be discussed with the patient because they offer the possibility of pregnancy and parenthood albeit with a non-genetic child. Mature oocyte collection requires ovarian stimulation. These oocytes can then either be frozen or else fertilized in vitro before freezing. These options are not open to all patients however. Ovarian stimulation takes a minimum of 2 weeks, and this delay is prohibitive for many patients with haematological malignancies. The requirement for partner or donor sperm for embryo cryopreservation is another potential drawback; for some patients, sperm is not available, and for others the involvement of a partner/sperm donor limits future reproductive autonomy as consent from the sperm provider must be given not only at the time of cryopreservation but also at the time of reimplantation.

\section{Ovarian Tissue Cryopreservation (OTC)}

OTC is considered experimental and is not available to all patients. Nonetheless, reports of pregnancy after OTC are increasing (Van der Ven et al. 2016), and it is the only option open to prepubertal patients or to women who cannot tolerate a significant delay in treatment due to disease severity or progression. Cortical fragments containing primordial follicles with immature oocytes can be obtained by laparoscopy and cryopreserved. Ideally, ovarian tissue should be obtained before the patient has been exposed to chemotherapy, but this is not always possible and is not an absolute requirement.

A major concern reimplanting cryopreserved ovarian tissue is the possibility of reseeding the tumour. The risk depends on the individual dis- ease. Assessment by PCR of ovarian tissue taken from patients with leukemia (CML, AML, ALL), tested positive for disease in a number of cases and assessment of tissue from mice with severe combined immunodeficiency confirmed, confirms the leukaemic potential of the tissue (Rosendahl et al. 2013). As a result, reintroduction of ovarian tissue from patients with leukemia would not currently be recommended. In the future, maturation in vitro of follicles from cryopreserved tissue may enable production of a viable disease-free alternative. In patients with lymphoma, histologically negative samples of ovarian issue have been transplanted without initiating relapse, but in some cases the follow-up time was short.

\subsubsection{Children and Adolescents}

Fertility preservation in children has been the subject of recent guidelines from the paediatric diseases working party of the EBMT (Dalle et al. 2017; Balduzzi et al. 2017). Extreme sensitivity is required, and parents have to be given complete information on the process, associated risks and success rates. For prepubertal girls, OTC is currently the only potential fertility-sparing option. In peri-pubertal boys, it is sometimes possible to extract sperm using surgery/microdissection or electroejaculation under general anaesthetic. In prepubertal males the only option is testicular tissue cryopreservation; although work in animal models is encouraging, there have been no reports to date of reimplanted testicular tissue leading to human live births.

\subsubsection{Management of Pregnancy After HSCT}

Most patients or their partners who conceive after HSCT have uncomplicated pregnancies. Chemoradiotherapy can potentially affect a variety of maternal organs relevant to a successful pregnancy outcome, for example, renal, cardiac and pulmonary toxicity. Patients at risk should have an expert medical review early in pregnancy and may require regular specialist monitoring throughout and review by an anaesthetist 
prior to delivery. Patients who have had TBI or pelvic irradiation have an increased risk of premature and small birth weight babies. Miscarriage rates are typically comparable to the background population, however, and no significant increase in congenital malformations or genetic abnormalities have so far been described when conception has taken place long after completion of therapy (Meirow and Schiff 2005; Green et al. 2009). Animal experiments suggest that most cytotoxic drugs are mutagenic and teratogenic to oocytes exposed during the maturation phase. In humans, this phase lasts approximately 6 months (Meirow and Schiff 2005), so there is a theoretical advantage to delaying conception for 6 months after completing gonadotoxic treatments.

\subsection{Sexual Function}

\subsubsection{Background}

Alterations in sexual function and sexual satisfaction are among the most common of all complications in long-term survivors of transplantation with $46 \%$ male and $80 \%$ female survivors describing sexual problems 5 years post transplant (Syrjala et al. 2008). Sexual complications in both allogeneic and autologous transplant recipients include changes in libido, dyspareunia (females) and erectile and ejaculatory dysfunction (males) (Li et al. 2015). Allogeneic recipients have additional problems linked to acute or chronic GVHD (Wong et al. 2013).

Sexuality is also affected, and this is multifactorial due to decreased self-confidence, stress, anxiety and fear of recurrence, together with a change in body image (Yi and Syrjala 2009). The sexual well-being of the survivor is also determined by their relationship with their partner (Langer et al. 2007). The partners of patients may experience a decrease in sexual desire and anxiety about initiating sexual activity with survivors. Poor communication can contribute to long-term sexual dysfunction between survivors and their partners (Hawkins et al. 2009).

\subsubsection{Prevention/Management}

It is important to identify relevant issues before problems with sexuality and intimacy become entrenched. It is recommended that healthcare professionals discuss sexual function with patients at 6 months, 1 year and at least annually thereafter (Majhail et al. 2012). Prompt attention should be paid to reversible risk factors such as hypogonadism or symptoms associated with urogenital GVHD so that appropriate referrals can be made to either gynaecologists or urologists. Some male patients benefit from the prescription of erectile dysfunction medication, and hormone replacement should be discussed with women prior to leaving hospital if onset of menopause is likely. Women with vaginal dryness may benefit from lubricants or topical oestrogens, and those with GVHD may benefit from topical steroids (Tirri et al. 2015). The role of vaginal dilators in preventing vaginal stenosis is not clear (Miles and Johnson 2014). It is recognized that psychological factors can play a large part in sexual dysfunction after transplant and this is relevant not only for the patient but also for the partner. For the patient it can be difficult to separate the psychological issues from coexisting physical problems, but many couples will benefit from review by a sex therapist.

\section{References}

Balduzzi A, Dalle JH, Jahnukainen K, et al. Fertility preservation issues in pediatric hematopoietic stem cell transplantation: practical approaches from the consensus of the Pediatric Diseases Working Party of the EBMT and the International BFM Study Group. Bone Marrow Transplant. 2017;52:1406-15.

Chen H, Li J, Cui T, Hu L. Adjuvant gonadotropinreleasing hormone analogues for the prevention of chemotherapy induced premature ovarian failure in premenopausal women. Cochrane Database Syst Rev. 2011;11:CD008018.

Chow EJ, Anderson L, Scott Baker K, et al. Late effects surveillance recommendations among survivors of childhood hematopoietic cell transplantation: a Children's Oncology Group Report. Biol Blood Marrow Transplant. 2016;22:782-95.

Cohen A, Rovelli A, Merlo DF, et al. Risk for secondary thyroid carcinoma after hematopoietic stem-cell transplantation: an EBMT late effects working party study. J Clin Oncol. 2007;25:2449-54. 
Dalle JH, Lucchini G, Balduzzi A, et al. State-of-theart fertility preservation in children and adolescents undergoing haematopoietic stem cell transplantation: a report on the expert meeting of the Paediatric Diseases Working Party (PDWP) of the European Society for Blood and Marrow Transplantation (EBMT). Bone Marrow Transplant. 2017;52:1029-35.

Fenig E, Mishaeli M, Kalish Y, Lishner M. Pregnancy and radiation. Cancer Treat Rev. 2001;27:1-7.

Green DM, Sklar CA, Boice JD Jr, et al. Ovarian failure and reproductive outcomes after childhood cancer treatment: results from the Childhood Cancer Survivor Study. J Clin Oncol. 2009;27:2374-81.

Hawkins Y, Ussher GE, et al. Changes in sexuality and intimacy after the diagnosis and treatment of cancer: the experience of partners in a sexual relationship with a person with cancer. Cancer Nurs. 2009;32:271-80.

Kalich-Philosoph L, Roness H, Carmely A, et al. Cyclophosphamide triggers follicle activation and "burnout"; AS101 prevents follicle loss and preserves fertility. Sci Transl Med. 2013;15:185ra62.

Langer SL, Rudd ME, Syrjala KL. Protective buffering and emotional desynchrony among spousal care givers. Health Psychol. 2007;26:635-43.

Laursen EM, Holm K, Brocks V, et al. Doppler assessment of flow velocity in the uterine artery during pubertal maturation. Ultrasound Obstet Gynecol. 1996;8:341-5.

Li Z, Mewawalla P, Stratton P, et al. Sexual health in hematopoietic stem cell transplant recipients. Cancer. 2015;121:4124-31.

Majhail NS, Rizzo D, Lee SJ, et al. Recommended screening and preventive practices for long-term survivors after hematopoietic cell transplantation. Biol Blood Marrow Transplant. 2012;18:348-71.

Meirow D, Nugent D. The effects of radiotherapy and chemotherapy on female reproduction. Hum Reprod Update. 2001;7:535-43.

Meirow D, Schiff E. Appraisal of chemotherapy effects on reproductive outcome according to animal studies and clinical data. J Natl Cancer Inst Monogr. 2005;34:21-5.

Meirow D. Reproduction post-chemotherapy in young cancer patients. Mol Cell Endocrinol. 2000;169:123-31.

Meistrich ML, van Beek MEAB. Radiation sensitivity of the human testis. Adv Radiat Biol. 1990;14:227-68.

Miles T, Johnson N. Vaginal dilator therapy for women receiving pelvic radiotherapy. Cochrane Database Syst Rev. 2014;9:CD007291.

Reulen RC, Zeegers MP, Wallace WHB, et al. Pregnancy outcomes among adult survivors of childhood cancer in the British childhood Cancer survivor study. Cancer Epidemiol Biomark Prev. 2009;18:2239-47.

Rosendahl M, Greve T, Anderson CY. The safety of transplanting cryopreserved ovarian tissue in cancer patients: a review of the literature. J Assist Reprod Genet. 2013;30:11-24.

Rovo A, Aljurf M, Chiodi S, et al. Ongoing graftversus-host disease is a risk factor for azoospermia after allogeneic hematopoietic stem cell transplantation: a survey of the Late Effects Working Party of the European Group for Blood and Marrow Transplantation. Haematologica. 2013;98:339-45.

Salooja N, Szydlo RM, Socie G, et al. Pregnancy outcomes after peripheral blood or bone marrow transplantation: a retrospective study. Lancet. 2001;358:271-6.

Sanders JE, Paul AH, Woolfrey AE, et al. Thyroid function following hematopoietic cell transplantation in children: 30 years' experience. Blood. 2009;113: 306-8.

Syrjala KL, Kurland BF, Abrams JR, et al. Sexual function changes during the 5 years after high-dose treatment and hematopoietic cell transplantation for malignancy, with case-matched controls at 5 years. Blood. 2008;111:989-96.

Tirri BF, Hausermann P, Bertz H, et al. Clinical guidelines for gynecologic care after hematopoietic SCT. Report from the international consensus project on clinical practice in chronic GVHD. Bone Marrow Transplant. 2015;50:3-9.

Van der Ven H, Liebenthron J, Beckmann M, FertiPROTEKT network, et al. Ninety-five orthotopic transplantations in 74 women of ovarian tissue after cytotoxic treatment in a fertility preservation network: tissue activity, pregnancy and delivery rates. Hum Reprod. 2016;31:2031-41.

Wallace WH, Thomson AB, Saran F, Kelsey TW. Predicting age of ovarian failure after radiation to a field that includes the ovaries. Int $\mathrm{J}$ Radiat Oncol Biol Phys. 2003;62:738-44.

Wallace WHB, Thomson AB, Kelsey TW. The radiosensitivity of the human oocyte. Hum Reprod. 2003;18:117-21.

Wong FL, Francisco L, Togawa K, et al. Longitudinal trajectory of sexual functioning after hematopoietic cell transplantation: impact of chronic graftversus-host disease and total body irradiation. Blood. 2013;122:3973-81.

Yi JC, Syrjala K. Sexuality after hematopoietic stem cell transplantation. Cancer J. 2009;15:57-64. 
Open Access This chapter is licensed under the terms of the Creative Commons Attribution 4.0 International License (http://creativecommons.org/licenses/by/4.0/), which permits use, sharing, adaptation, distribution and reproduction in any medium or format, as long as you give appropriate credit to the original author(s) and the source, provide a link to the Creative Commons license and indicate if changes were made.

The images or other third party material in this chapter are included in the chapter's Creative Commons license, unless indicated otherwise in a credit line to the material. If material is not included in the chapter's Creative Commons license and your intended use is not permitted by statutory regulation or exceeds the permitted use, you will need to obtain permission directly from the copyright holder.

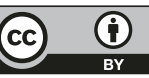

